\title{
Coronary calcification: Achilles' heel in the assessment for coronary artery disease in patients with symptomatic angina?
}

\author{
John N. Makaryus • Amgad N. Makaryus
}

Received: 29 October 2009/Accepted: 2 November 2009/Published online: 24 November 2009

(C) Springer Science+Business Media, B.V. 2009

Coronary calcium quantification has emerged as a reliable and independent predictor of adverse cardiovascular events. Advances in multidetector scanning and imaging protocols have resulted in decreased contrast volumes, shorter scan times, and decreased radiation exposure, as well as improved spatial resolution and enhanced image quality. In subsets of patients with high calcium scores (particularly $\geq 400$ ), however, the artifactual effects and scatter associated with radiodense calcium deposits have limited the specificity of CT coronary angiography (CTCA) in delineating the extent of clinically significant coronary artery disease (CAD). In the present study in the International Journal of Cardiovascular Imaging, Meijs et al. [1] conduct a multicenter prospective analysis of the diagnostic performance of 64-CTCA to predict the incidence of CAD in 360 patients who had been referred for conventional invasive coronary angiography (ICA) on the basis of symptomatic angina (stable and unstable) regardless of coronary calcium score (CS) over a 2-year time interval. As expected, the incidence of significant CAD correlated with increased CS. In patients with a CS $\geq 400$, the prevalence of significant CAD was $87 \%$ as compared

Editorial comment on the article of Meijs et al. (doi:10.1007/s10554-009-9485-7).

J. N. Makaryus · A. N. Makaryus ( $₫)$

North Shore University Hospital, Manhasset, NY, USA

e-mail: amakaryus@gmail.com to $24 \%$ in those with a $\mathrm{CS}<10$. The specificity of CTCA as well as the positive likelihood ratio for significant CAD decreased significantly, however, as the CS increased. In patients with a CS $<10$, the specificity was $89 \%$ (95\%CI 76-96\%) while in patients with $\mathrm{CS}>400$, the specificity was only $20 \%$ (95\% CI 5-49\%) [1].

The findings of this study are not inconsistent with the current literature, though the dramatic decline in specificity with increasing CS demonstrates a significant degree of inter-study variability. Early studies comparing the diagnostic capabilities of CTCA with ICA often excluded patients with elevated baseline $\mathrm{CS}$ as a result of the negative impact of high CS on CTCA accuracy or the inability to assess the presence or absence of stenosis with increased calcification [24]. Studies which did include arterial segments with high levels of calcification usually denoted a significant decline in the diagnostic capability of the test. For example, the multicenter ACCURACY (Assessment by Coronary Computed Tomographic Angiography of Individuals Undergoing Invasive Coronary Angiography) trial which enrolled 230 patients regardless of CS, demonstrated a decline of specificity of CTCA for the $50 \%$ threshold CAD level from $86.3 \%$ in patients with CS $\leq 400$ to $52.6 \%$ in patients with CS $>400(P<0.0003)$ [5].

Studies of the implications of reduced specificity of CTCA have suggested that patients with very high calcium scores should forego multidetector coronary CT angiography in favor of ICA given the decreased 
accuracy of the test with increased CS and the likelihood that individuals with very high CS have obstructive disease and will require invasive angiography anyway [6]. Severe coronary calcification impedes the ability to adequately assess the degree of lumen compromise and has repeatedly been shown to limit the test's diagnostic capability [7-9]. Given the lack of additional information likely to be gleaned from CTCA in both patients with a high pretest probability as well as those with a very low pretest probability of coronary artery disease, the test remains most useful primarily in patients with an intermediate likelihood of cardiovascular disease and symptoms potentially attributable to CAD [10]. Hence, the necessity of the test in high-risk individuals with symptomatic angina (and thus high pretest probability of coronary disease) is difficult to justify on the basis of current data, notwithstanding occasional data which may indicate minimal or no effect of CS on the specificity of the test [11]. This acknowledgement is particularly relevant given the low probability that CTCA will preclude invasive angiography in these patients. This philosophy is reflected in the joint appropriateness criteria for cardiac CT published in 2006 [12].

The limited specificity of CTCA in detecting clinically relevant coronary artery stenoses in patients with high CS correlates with the recent trend to focus upon improving visualization of noncalcified, lipidladen/mixed lesions rather than relying solely upon calcium scoring to estimate the risk of adverse cardiovascular disease events. The recently published Multi-Ethnic Study of Atherosclerosis (MESA) trial emphasized that even asymptomatic individuals with very low CS (1-10) may be at risk of significant cardiovascular disease events compared to individuals with a CS of 0 [13]. In addition, 13-18\% of coronary arteries with $\geq 75 \%$ stenosis had a CS of 0 , raising further concerns about relying too heavily upon calcium scoring [14].

At our institution, we retrospectively determined that despite a relatively equivalent likelihood of cardiovascular disease events between males and postmenopausal females in an age and risk-factor matched cohort, females had significantly lower calcium scores in each of the four major coronary vessels reflecting the importance of lipid-laden and mixed plaques in leading to CAD events [15].
The study by Meijs et al. [1] solidifies an accepted concept in the field of cardiac computed tomography; namely, that the test is extremely useful when used under the appropriate clinical circumstances. While constantly improving technology with enhanced temporal and spatial resolution as well as newer study protocols have successfully minimized the reduction of diagnostic performance attributable to motion artifacts, increasing degrees of coronary calcification may still limit the specificity of the test in high-risk individuals. Thus, while the future is promising and the number of appropriate indications for CTCA is likely to increase, current technology still dictates that the test should primarily be reserved for intermediaterisk individuals in whom a positive or negative scan may alter clinical pathways and CTCA may serve as a true "gateway" for invasive angiography.

\section{References}

1. Meijs M, Meijboom WB, Prokop M, Mollet NR, van Mieghem CA, Doevendans PA, de Feyter PJ, Cramer MJ (2009) Is there a role for CT coronary angiography in patients with symptomatic angina? Effect of coronary calcium score on identification of stenosis. Int J Cardiovasc Imaging. doi:10.1007/s10554-009-9485-7

2. Raff GL, Gallagher MJ, O’Neill WW, Goldstein JA (2005) Diagnostic accuracy of noninvasive coronary angiography using 64-slice spiral computed tomography. J Am Coll Cardiol 46(3):552-557

3. Garcia MJ, Lessick J, Hoffmann MHK, for the CATSCAN Study Investigators (2006) Accuracy of 16-row multidetector computed tomography for the assessment of coronary artery stenosis. JAMA 296(4):403-411

4. Miller JM, Rochitte CE, Dewey M, Arbab-Zadeh A, Niinuma H, Gottlieb I, Paul N, Clouse ME, Shapiro EP, Hoe J, Lardo AC, Bush DE, de Roos A, Cox C, Brinker J, Lima JA (2008) Diagnostic performance of coronary angiography by 64-row CT. N Engl J Med 359(22):2324-2336

5. Budoff MJ, Dowe D, Jollis JG, Gitter M, Sutherland J, Halamert E, Scherer M, Bellinger R, Martin A, Benton R, Delago A, Min JK (2008) Diagnostic performance of 64multidetector row coronary computed tomographic angiography for evaluation of coronary artery stenosis in individuals without known coronary artery disease: results from the prospective multicenter ACCURACY (assessment by coronary computed tomographic angiography of individuals undergoing invasive coronary angiography) trial. J Am Coll Cardiol 52(21):1724-1732

6. Kuettner A, Beck T, Drosch T, Kettering K, Heuschmid M, Burgstahler C, Claussen CD, Kopp AF, Schroeder S (2005) Image quality and diagnostic accuracy of non-invasive coronary imaging with 16 detector slice spiral computed 
tomography with $188 \mathrm{~ms}$ temporal resolution. Heart 91(7): 938-941

7. Kuettner A, Kopp A, Schroeder S, Rieger T, Brunn J, Meisner C, Heuschmid M, Trabold T, Burgstahler C, Martensen J (2004) Diagnostic accuracy of multidetector computed tomography coronary angiography in patients with angiographically proven coronary artery disease. J Am Coll Cardiol 43(5):831-839

8. Budoff MJ, McClelland RL, Nasir K, Greenland P, Kronmal RA, Kondos GT, Shea S, Lima JA, Blumenthal RS (2009) Cardiovascular events with absent or minimal coronary calcification: the multi-ethnic study of atherosclerosis (MESA). Am Heart J 158(4):554-561

9. Hoffmann U, Moselewski F, Cury RC, Ferencik M, Jang IK, Diaz LJ, Abbara S, Brady TJ, Achenbach S (2004) Predictive value of 16-slice multidetector spiral computed tomography to detect significant obstructive coronary artery disease in patients at high risk for coronary artery disease: patient-versus segment-based analysis. Circulation 110(17):2638-2643

10. Ong TK, Chin SP, Liew CK, Chan WL, Seyfarth MT, Liew HB, Rapaee A, Fong YY, Ang CK, Sim KH (2006) Accuracy of 64-row multidetector computed tomography in detecting coronary artery disease in 134 symptomatic patients: influence of calcification. Am Heart J 151:13231326

11. Pundziute G, Schuijf JD, Jukema JW, Lamb HJ, de Roos A, van der Wall EE, Bax JJ (2007) Impact of coronary calcium score on diagnostic accuracy of multislice computed tomography coronary angiography for detection of coronary artery disease. J Nucl Cardiol 14:36-43

12. Rosen BD, Fernandes V, McClelland RL, Carr JJ, Detrano R, Bluemke DA, Lima JA (2009) Relationship between baseline coronary calcium score and demonstration of coronary artery stenoses during follow-up MESA (multiethnic study of atherosclerosis). JACC Cardiovasc Imaging 2(10): 1175-1183

13. Schroeder S, Achenbach S, Bengel F, Burgstahler C, Cademartiri F, de Feyter P, George R, Kaufmann P, Kopp AF, Knuuti J, Ropers D, Schuijf J, Tops LF, Bax JJ (2008) Cardiac computed tomography: indications, applications, limitations, and training requirements: report of a Writing Group deployed by the Working Group Nuclear Cardiology and Cardiac CT of the European Society of Cardiology and the European Council of Nuclear Cardiology. Eur Heart J 29(4):531-556

14. Hendel RC, Patel MR, Kramer CM, Poon M, Hendel RC, Carr JC, Gerstad NA, Gillam LD, Hodgson JM, Kim RJ, Kramer CM, Lesser JR, Martin ET, Messer JV, Redberg RF, Rubin GD, Rumsfeld JS, Taylor AJ, Weigold WG, Woodard PK, Brindis RG, Hendel RC, Douglas PS, Peterson ED, Wolk MJ, Allen JM, Patel MR (2006) ACCF/ ACR/SCCT/SCMR/ASNC/NASCI/SCAI/SIR 2006 appropriateness criteria for cardiac computed tomography and cardiac magnetic resonance imaging: a report of the American College of Cardiology Foundation Quality Strategic Directions Committee Appropriateness Criteria Working Group, American College of Radiology, Society of Cardiovascular Computed Tomography, Society for Cardiovascular Magnetic Resonance, American Society of Nuclear Cardiology, North American Society for Cardiac Imaging, Society for Cardiovascular Angiography and Interventions, and Society of Interventional Radiology. J Am Coll Cardiol 48:1475-1497

15. Makaryus JN, Makaryus AN, Tai JY, Doshi JV, Boxt LM (2008) The implications of gender difference in coronary calcification as assessed by 64-CT coronary angiography. J Cardiovasc Comput Tomogr 2(4S):S44 